\title{
Criminologie
}

\section{Un exemple de justice réparatrice au Québec : la médiation et les organismes de justice alternative}

\section{Serge Charbonneau et Denis Béliveau}

Volume 32, numéro 1, printemps 1999

La justice réparatrice

URI : https://id.erudit.org/iderudit/004711ar

DOI : https://doi.org/10.7202/004711ar

Aller au sommaire du numéro

\section{Éditeur(s)}

Les Presses de l'Université de Montréal

ISSN

0316-0041 (imprimé)

1492-1367 (numérique)

Découvrir la revue

Citer cet article

Charbonneau, S. \& Béliveau, D. (1999). Un exemple de justice réparatrice au Québec : la médiation et les organismes de justice alternative. Criminologie, 32(1), 57-77. https://doi.org/10.7202/004711ar
Résumé de l'article

Depuis une vingtaine d'années, le secteur de la justice des mineurs au Québec a connu de nombreux bouleversements. Cette période a notamment été marquée par la tenue de plusieurs commissions d'étude ainsi que par l'adoption de la Loi sur la protection de la jeunesse et de la Loi sur les jeunes contrevenants. Elle se caractérise également par la mise en place des premières expériences de justice réparatrice reposant sur la médiation. Dans cet article, les auteurs cherchent d'abord à situer dans quel contexte s'est développée la médiation au Québec, depuis la mise sur pied du Projet d'intervention jeunesse en 1977 jusqu'à la création des premiers organismes de justice alternative. En se penchant sur les raisons pouvant expliquer le lent développement de la médiation au Québec, les auteurs examinent notamment l'hypothèse qui met en cause le modèle protectionnel et sa prééminence dans les institutions pour mineurs. Une telle dynamique, qui aurait pour effet d'amener les professionnels à concentrer leur attention sur le développement des jeunes au détriment des besoins des victimes d'actes criminels, expliquerait le recours peu fréquent à la médiation. Les auteurs se penchent ensuite sur l'évolution des pratiques de médiation dans les organismes de justice alternative. Ils montrent que les façons de faire ont connu d'importantes modifications, lesquelles sont à mettre au compte de l'influence de différentes perspectives européennes et américaines. Ainsi, les débats sur le potentiel réparateur de la médiation, loin d'être terminés, animent encore le milieu et soulèvent d'importantes questions. Plus que jamais, la nécessité de recherches descriptives et évaluatives sur le potentiel de la médiation en matière criminelle se fait donc sentir.
Tous droits réservés @ Les Presses de l'Université de Montréal, 1999
Ce document est protégé par la loi sur le droit d'auteur. L’utilisation des services d'Érudit (y compris la reproduction) est assujettie à sa politique d'utilisation que vous pouvez consulter en ligne.

https://apropos.erudit.org/fr/usagers/politique-dutilisation/ 


\section{Un exemple de justice réparatrice au Québec : la médiation et les organismes de justice alternative}

Serge Charbonneau

coordonnateur

Regroupement des organismes de justice alternative du Québec (ROJAQ)

scharbonneau@rojaq.qc.ca

Denis Béliveau

Chercheur autonome et consultant auprès d'organisations communautaires denisbel@total.net

RÉsumÉ Depuis une vingtaine d'années, le secteur de la justice des mineurs au Québec a connu de nombreux bouleversements. Cette période a notamment été marquée par la tenue de plusieurs commissions d'étude ainsi que par l'adoption de la Loi sur la protection de la jeunesse et de la Loi sur les jeunes contrevenants. Elle se caractérise également par la mise en place des premières expériences de justice réparatrice reposant sur la médiation. Dans cet article, les auteurs cherchent d'abord à situer dans quel contexte s'est développée la médiation au Québec, depuis la mise sur pied du Projet d'intervention jeunesse en 1977 jusqu'à la création des premiers organismes de justice alternative. En se penchant sur les raisons pouvant expliquer le lent développement de la médiation au Québec, les auteurs examinent notamment l'hypothèse qui met en cause le modèle protectionnel et sa prééminence dans les institutions pour mineurs. Une telle dynamique, qui aurait pour effet d'amener les professionnels à concentrer leur attention sur le développement des jeunes au détriment des besoins des victimes d'actes criminels, expliquerait le recours peu fréquent à la médiation. Les auteurs se penchent ensuite sur l'évolution des pratiques de médiation dans les organismes de justice alternative. Ils montrent que les façons de faire ont connu d'importantes modifications, lesquelles sont à mettre au compte de l'influence de différentes perspectives européennes et américaines. Ainsi, les débats sur le potentiel réparateur de la médiation, loin d'être terminés, animent encore le milieu et soulèvent d'importantes questions. Plus que jamais, la nécessité de recherches descriptives et évaluatives sur le potentiel de la médiation en matière criminelle se fait donc sentir.

Criminologie, vol. 32, n 1 (1999) 
ABSTRACT In the last 20 years, juvenile justice in Quebec has undergone many changes. This period has been caracterised by numerous Commissions of Inquiry and by the adoption of the Youth Protection Act and the Young Offenders Act. It is also marked by the implementation of the first attempts at restorative justice based on mediation. In the following article, the authors first place the development of mediation in Quebec in its context, from the implementation of "Projet d'intervention jeunesse" in 1977 through to the creation of the first alternative justice organisms. Among the reasons explaining the slow development of mediation in Quebec, the authors study the hypothesis that identifies the pre-eminence of the protectionnist model in juvenile institutions as a cause. The under use of mediation could be explained by the professional attention given to youth development, which neglects the victim's needs. Second, the authors study the evolution of mediation practices in alternative justice organisms. They demonstrate that mediation practices have been significantly modified in response to different European and American perspectives. The transformative potential of mediation still raises important questions about the principal aim of mediation. Experts still often debate the real impact of mediation. More than ever, there is a need for descriptive and evaluative research on the potential of mediation in criminal matters.

\section{Introduction}

Se définissant comme une nouvelle façon de percevoir et de réagir à la criminalité, la justice réparatrice s'impose progressivement dans un nombre grandissant de pays. Par ses qualités, la médiation constitue l'une des pratiques les plus fréquemment associées à ce mode de justice. En ce qui concerne la médiation en matière criminelle, plusieurs auteurs s'entendent pour dire que les premières expériences canadiennes ont eu lieu en 1974 dans la ville de Kitchener en Ontario (Umbreit, 1995 ; Wright, 1996 ; Faget, 1997a). Au Québec, différentes expériences se sont inspirées d'un tel modèle.

Il y a près de vingt ans, naissait à Montréal un organisme communautaire se donnant pour objectif d'offrir aux jeunes contrevenants la possibilité de réparer leurs méfaits tout en évitant le processus judiciaire. Cette première expérience en matière de mesures alternatives à la judiciarisation marque le point de départ d'un mouvement qui va rapidement faire boule de neige au Québec. Connues aujourd'hui sous le nom d'organismes de justice alternative $(\mathrm{OJA})$, près d'une quarantaine d'organisations interviennent auprès de jeunes contrevenants en leur proposant un vaste éventail de mesures, au nombre desquelles figure la médiation. 
Le but de cet article est de présenter l'histoire de ce mouvement tout en apportant une attention particulière à la place qu'y occupe la médiation. Il vise plus particulièrement à mettre en lumière une pratique encore peu connue ${ }^{1}$ tout en proposant une réflexion critique au sujet des grands débats touchant aux objectifs de la médiation comme forme de justice réparatrice.

\section{Vers la création des 0JA}

La prise de conscience à l'effet que l'appareil judiciaire se doit de réserver un traitement particulier aux jeunes, que ce soit en termes de protection, de traitement ou de punition, remonte déjà à plus d'un siècle. Cette reconnaissance s'inscrit dans un mouvement plus large, relevant à la fois de l'évolution des rapports sociaux, des structures familiales, des modèles d'intervention et des représentations que l'on se fait de l'enfance. À l'instar de la plupart des pays occidentaux, le Canada se dote, à partir de la seconde moitié du XIX ${ }^{\mathrm{e}}$ siècle, de lois et d'institutions spécifiques pour le traitement des mineurs (Trépanier et Tulkens, 1995). Une première loi est votée en 1857 ; son adoption témoigne d'un début de préoccupation à l'égard du traitement à réserver aux jeunes (D'Amours, 1982). On souhaite alors accélérer les procédures qui encadrent la tenue des procès des jeunes, afin de leur éviter un emprisonnement trop long ${ }^{2}$.

Quelques années plus tard (1869), le gouvernement procède à la création des premières institutions spécialement conçues pour la réhabilitation des jeunes (Écoles industrielles et Écoles de réforme). L'appareil judiciaire acquiert alors une nouvelle fonction. Il ne s'agit plus simplement d'isoler les jeunes afin de les punir, mais plutôt de les placer dans des institutions où l'on pourra les réhabiliter. Puis vint la loi de 1908 sur les jeunes délinquants. Cette loi participe d'une même logique et va même plus loin en faisant de la réhabilitation le principe directeur devant guider le traitement

I. La revue Porte ouverte a récemment consacré un numéro spécial à la justice réparatrice. Les responsables de ce numéro sont dans l'erreur en laissant entendre que cette forme de justice n'existe pas au Québec. Voir ASRSQ, 1998.

2. Pour la première fois en outre, la délinquance juvénile fait l'objet d'une définition légale : est considérée jeune délinquant toute personne âgée de moins de 16 ans coupable d'avoir « commis ou cherché à commettre, ou d'avoir aidé, favorisé, conseillé ou procuré les moyens de commettre une offense [...] considérée ou déclarée simple larcin [...]». Acte pour accélérer le procès et la punition des jeunes délinquants. Statuts de la province du Canada, 20 Victoria, chap. XXIX, article I, 1857. 
à donner aux jeunes aux prises avec la justice. Elle comporte en effet un principe important, à savoir que le jeune délinquant ne doit pas être considéré comme un criminel, mais bien comme un individu qui, à l'instar de l'enfant maltraité ou négligé, a besoin de correction, de soin et de surveillance (Trépanier, 1988). Entre l'adoption de cette loi et la Révolution tranquille, l'approche éducative s'impose sur le traitement pénitentiaire, le recours à la prison cédant sa place au placement dans une institution spécialisée ou à la liberté surveillée chez un tiers.

Au Québec comme ailleurs, les années 1960 se caractérisent par de rapides et profondes transformations. L'effervescence qui règne alors favorise une plus grande mobilisation politique et sociale. Des groupes porteurs de revendications sociales émergent, les syndicats se radicalisent et les groupes communautaires s'unissent pour exiger des transformations. En prenant le virage de la laïcisation et de la modernisation, l'État québécois entend revoir en profondeur le fonctionnement de la fonction publique, de l'éducation, de la santé et des services sociaux. Le secteur de la justice n'y échappe pas et les gouvernements (à Québec comme à Ottawa) s'engagent dans de vastes consultations sur l'appareil judiciaire et les prisons, l'objectif étant de les transformer et de les humaniser.

Les professionnels des sciences sociales formés dans les années 1960 et 1970 participent d'une façon active aux débats au sujet de la justice et des modèles d'intervention à privilégier. Invités à prendre part aux commissions d'enquête alors mises sur pied, ils font part des résultats de leurs travaux et de leurs recommandations, mais aussi de leurs interrogations face à l'administration du système pénal, accélérant du même coup un certain nombre de transformations. Cette période est marquée, il est vrai, par l'émergence d'un courant très critique en criminologie et dans les autres sciences sociales. De nombreux ouvrages (comme ceux de Becker, Goffman, Schurr et Foucault) remettent en question les façons traditionnelles de définir le crime, la délinquance ou la déviance, démontrant que ces catégories ne sont pas nécessairement données en soi, questionnant du même coup la légitimité des modèles dominants d'intervention et favorisant la recherche de nouveaux modes d'intervention, moins centrés sur le « délinquant » que sur son environnement ou sur les préjudices causés par ses comportements.

\section{Des alternatives à l'emprisonnement}

Historiquement, le concept de justice réparatrice s'est développé à partir des critiques émises à l'égard du système pénal et de son administration 
(Desdevises, 1993; Tremblay, 1994). Aux côtés des objectifs du modèle punitif (rétablir l'ordre moral) ou réhabilitatif (rééduquer), émerge peu à peu l'idée que l'intervention doit d'abord permettre la réduction et la réparation des torts (Walgrave, 1993). Un tel concept est en outre indissociable d'un mouvement de fond en faveur de la non-judiciarisation ou de la déjudiciarisation des conflits. Bien que présent dans d'autres pays, l'intérêt pour les mécanismes alternatifs de règlements des conflits (MARC ${ }^{3}$ ) s'est plus particulièrement développé aux États-Unis. Au cours des années 1970, plusieurs expériences y sont menées afin d'élaborer des structures permettant de régler certains comportements sans avoir recours à l'appareil judiciaire. Ces expériences sont souvent désignées sous l'abréviation de VORP (Victim Offender Reconciliation Program) (Zehr, 1990 ; Umbreit et Greenwood, 1998). L'ensemble de ces initiatives ont en commun de proposer des alternatives aux sanctions traditionnelles : le travail communautaire, la conciliation directe avec la victime, le remboursement, etc., sont au nombre des solutions envisagées.

Peu à peu, ce type de programmes se développe au Canada. En 1976, la Commission de réforme du droit endosse le modèle de la réparation directe, reconnaissant « cette idée fondamentale qu'en faisant quelque chose pour autrui, le délinquant dédommage la société » (CRD, 1976 : 147). Les programmes s'inspirant du modèle de justice réparatrice bénéficient en outre d'un accueil favorable parmi les groupes communautaires qui voient d'un bon œil de telles initiatives, reconnaissant qu'elles parviennent à concilier avantageusement les intérêts de la société et de la victime au traitement juste et équitable du « délinquant ». À Montréal, le Bureau de consultation jeunesse (BCJ), un organisme communautaire d'assistance et d'accompagnement, accepte de parrainer un projet de déjudiciarisation auprès des jeunes contrevenants. Connue sous le nom de « Projet Intervention Jeunesse ", cette expérience se déroule entre les mois de mars 1977 et d'octobre 1979 sur le territoire d'Outremont. Les intervenants du $\mathrm{BCJ}$, en collaboration avec une équipe de policiers-jeunesse, élaborent alors un protocole visant à soustraire les jeunes du processus judiciaire en leur proposant des solutions de rechange (Charbonneau, 1998b). Cellesci prennent alors la forme d'une rencontre de médiation (ou de conciliation avec la victime), d'une lettre d'excuse à rédiger, d'une participation à des travaux bénévoles au profit de la victime ou de la communauté, ou encore d'une mesure de « conscientisation » par une intervention de type

3. Les MARC étant la traduction des ADR ou Alternative Dispute Resolution. 
counselling (Lajoie, 1979). Cette première expérience en matière de mesures alternatives à la judiciarisation marque un tournant et servira de modèle aux autres initiatives de déjudiciarisation.

La fin de cette expérience coïncide en outre avec l'adoption de la Loi sur la protection de la jeunesse (Loi 24), en vigueur à compter du 15 janvier 1979. Si les échanges en Commission parlementaire ont soulevé des débats importants, on remarque que l'ensemble des intervenants et des parlementaires souscrivent alors aux objectifs de cette loi : favoriser la déjudiciarisation, rendre plus fonctionnels et opérationnels les réseaux de la justice et des affaires sociales et, finalement, limiter le développement bureaucratique de l'État ${ }^{4}$. En fait, cette loi est d'une portée plus large, redéfinissant les modalités d'intervention en termes de protection de la jeunesse (article 38), tout en visant les adolescents aux prises avec des problèmes de délinquance (article 40). Centrée sur le respect des droits des adolescents, la Loi 24 prône une intervention personnalisée et le maintien des jeunes, autant que faire se peut, dans leur milieu naturel de vie. À cette fin, elle introduit un nouveau concept : les mesures volontaires. Il s'agit alors d'offrir aux jeunes, aux prises avec la justice, la possibilité de s'amender en effectuant, par exemple, un certain nombre d'heures de travaux communautaires. Encouragés par l'expérience menée par le BCJ et en s'appuyant sur certaines dispositions de la Loi 24 , différents groupes mettent en place des structures leur permettant d'expérimenter un modèle alternatif d'intervention.

\section{Les OJA et la Loi sur les jeunes contrevenants}

L'expérience menée par le BCJ sur le territoire d'Outremont incite d'abord deux étudiants de l'École de criminologie de l'Université de Montréal à élaborer un projet semblable qui démarre en juin 1980. Le « Programme de travaux communautaires » de Montréal vise à permettre à des adolescents ayant commis une infraction de réparer leur geste en prenant conscience du préjudice causé par leurs comportements. En plus d'obtenir réparation, la communauté d'attache de ces jeunes est indirectement mise à contribution dans cette démarche, en ce que ces derniers doivent alors travailler auprès d'un organisme public ou à but non lucratif.

Pendant que se déroulait cette expérience, d'autres projets similaires voient le jour ailleurs au Québec. C'est notamment le cas à Victoriaville, à Québec, à Gatineau puis à Trois-Rivières. Dans le sillage de ces

4. Journal des débats, vol. 19, n 123 . Débats du 24 novembre 1977. 
premiers organismes, d'autres groupes se forment un peu partout au Québec. Aux sept organismes actifs en 1983, s'ajoutent dix-sept autres en avril 1985. Depuis, leur nombre a encore augmenté, si bien qu'on en dénombre aujourd'hui près d'une quarantaine, répartis dans l'ensemble des régions administratives du Québec ${ }^{5}$.

La législation concernant la justice des mineurs connaît alors d'importants changements. Le gouvernement fédéral décide en effet de revoir la Loi de 1908, devenue désuète. C'est ainsi qu'une nouvelle loi en matière de justice pour mineurs est promulguée le 2 avril 1984. Le libellé même du titre de la nouvelle loi témoigne déjà d'une évolution dans l'approche : il n'est plus question d'une loi concernant les jeunes délinquants, mais plutôt d'une Loi sur les jeunes contrevenants (LJC). Cette loi vise tous les adolescents âgés de 12 à 17 ans inclusivement soupçonnés d'avoir commis une infraction au Code criminel ou à d'autres lois et règlements fédéraux. Tout en reconnaissant que ces derniers peuvent avoir des besoins qui leur sont propres, la LJC s'articule autour de principes qui tendent à établir un équilibre entre les droits et les responsabilités des adolescents, et ceux de la société. Elle s'inscrit dans la foulée des expériences de déjudiciarisation en ce qu'elle présente des « dispositions innovatrices qui permettent de substituer, pour les infractions moins graves, des mesures de rechange à la procédure judiciaire officielle » (Solliciteur général du Canada, 1982 : 10). La LJC permet donc le développement de mesures de réparation des torts causés, devenant ainsi une des premières lois à favoriser le dédommagement direct à la victime sous toutes ses formes.

En pratique, le dossier d'un jeune contrevenant est d'abord remis au substitut du procureur général qui effectue un premier tri. Trois possibilités s'offrent à ce dernier : a) il peut fermer le dossier ; b) il peut le diriger vers un délégué à la jeunesse qui va rencontrer le jeune et lui offrir de participer à une «mesure de rechange »; c) il peut traduire le jeune devant la Chambre de la jeunesse où il y aura procès. Si le jeune est reconnu coupable, le juge prononce une "mesure ordonnée ». Pour voir à l'encadrement des mesures de rechange et de certaines mesures ordonnées, la Loi prévoit la possibilité de faire appel à des organismes communautaires. Bénéficiant déjà d'une expertise en ce domaine, les OJA se voient dès lors chargés d'encadrer un certain nombre de ces jeunes et de les accompagner dans une démarche de réparation.

5. Depuis leur création, ils ont été désignés de différentes façons : jusqu'en 1986, ils étaient connus sous le nom d' « organismes référents » puis, jusqu'en 1996, sous le vocable d'« organismes orienteurs » et, depuis cette date, par celui d'« organismes de justice alternative». 


\section{Les mesures offertes dans les OJA}

Comme en témoigne le tableau 1, les OJA profitent de cette reconnaissance et connaissent un essor rapide à compter de la seconde moitié des années 1980. En 1984-1985, une dizaine d'organismes sont déjà en activité. Ils accueillent alors un peu plus de 700 jeunes. Avec la création de nouveaux organismes, le nombre de jeunes reçus par l'ensemble des OJA s'élève déjà à 4841 en 1987-1988. Leur nombre a pratiquement triplé depuis, pour atteindre 12998 en 1997-1998.

Au fil des années, les OJA ont cherché à développer un modèle d'intervention visant à permettre à des jeunes ayant commis un geste répréhensible de ne pas avoir à se présenter devant le tribunal ou, le cas échéant, de pouvoir s'amender sous la supervision d'une tierce personne de sa communauté. Les pratiques d'intervention des OJA se sont ainsi diversifiées et comprennent aujourd'hui différentes mesures.

Les premiers organismes ont d'abord construit leur expertise en offrant aux jeunes la possibilité d'effectuer des travaux communautaires afin de réparer les torts qu'ont engendrés leurs comportements. On parle ici de réparation symbolique (ou indirecte) dans la mesure ou le travail ne bénéficie pas directement à la personne qui a été victime, mais à la collectivité proche du jeune.

Aux côtés des travaux communautaires, différents outils visant la responsabilisation des jeunes ont été développés. La mesure A.A.S. (pour " amélioration des aptitudes sociales ») peut prendre différentes formes. Le plus souvent, les adolescents sont invités à prendre part à des activités de créativité et d'apprentissage, à des groupes d'entraide, ou encore à des séances d'information sur des sujets bien précis (drogue, alcool, violence, vol à l'étalage). D'autres vont participer à des activités de loisirs ou à l'organisation d'un projet (par exemple : nettoyage d'un parc, bénévolat lors d'une fête de quartier...).

Préoccupés par le peu de place faite aux victimes, d'autres organismes ont été amenés au fil des ans à offrir un service de médiation et de conciliation. Cette mesure vise ici à déterminer une façon par laquelle le jeune qui a commis un geste répréhensible peut réparer, et ce, à la satisfaction des deux parties. Cette démarche peut se faire en présence d'une tierce personne (médiation) ou sans rencontre directe entre les parties (conciliation). À l'exception d'un groupe d'intervenants associé au YMCA de Montréal qui, dès 1982, a mis sur pied un programme de médiation (programme « Entente»), les premiers OJA n'offraient pas cette mesure 
TABLEAU 1

Évolution du nombre de jeunes accueillis dans les 0JA, 1984-1985 à 1997-1998

\begin{tabular}{|cc|cc|}
\hline Année & Nombre de jeunes & Année & Nombre de jeunes \\
\hline $1984-1985$ & 723 & $1991-1992$ & 9045 \\
$1985-1986$ & 3304 & $1992-1993$ & 10366 \\
$1986-1987$ & 4077 & $1993-1994$ & 10967 \\
$1987-1988$ & 4841 & $1994-1995$ & 10638 \\
$1988-1989$ & 5394 & $1995-1996$ & 12213 \\
$1989-1990$ & 6211 & $1996-1997$ & 12307 \\
$1990-1991$ & 7008 & $1997-1998$ & 12998 \\
\hline
\end{tabular}

(Chamberlain, 1987). Quelques années plus tard, deux autres OJA (ceux de Trois-Rivières et de Gatineau) élaborent un programme de médiation. Entre 1988 et 1993, ils sont pratiquement les seuls OJA du Québec à pratiquer la médiation. Ailleurs, l'administration de cette mesure relevait de la responsabilité des délégués à la jeunesse.

Par ailleurs, le Code de procédure pénale (C.P.P.) a également été adapté à la situation des mineurs qui peuvent être poursuivis en vertu de cette loi. Des procédures relatives à la constatation, à la répression et à la sanction pénale des infractions créées par la législation et la réglementation québécoises (telles que les infractions au Code de sécurité routière, aux règlements municipaux relatifs aux parcs, au stationnement, à la paix publique, etc.) sont donc prévues. Sur cette base, certains des jeunes qui sont reçus dans un OJA ont à effectuer des travaux compensatoires, le nombre d'heures variant en fonction de l'importance de l'amende à payer.

\section{Le développement de la mesure de médiation dans les 0JA}

Comme l'illustre le tableau 2, les travaux communautaires constituent depuis longtemps la principale mesure administrée par les OJA. La médiation ne constitue pas encore une mesure très fréquente. Pourtant, dès 1984, les auteurs de la Loi sur les jeunes contrevenants et du programme de mesures de rechange l'avaient identifiée comme l'une des mesures possibles à offrir aux jeunes.

De fait, cette mesure a connu un très lent développement dans les OJA. Jusqu'en 1996-1997, elle ne représente qu'une très faible proportion 
TA BLEA U 2

Type et évolution de mesures administrées par les 0JA, 1991-1992 à 1997-1998 (en nombre absolu de mesures)

\begin{tabular}{|c|c|c|c|c|c|c|}
\hline Année & $\begin{array}{l}\text { Travaux } \\
\text { communau- } \\
\text { taires }\end{array}$ & $\begin{array}{c}\text { Amélior. } \\
\text { des } \\
\text { aptitudes } \\
\text { sociales } \\
\text { (A.A.S.) }\end{array}$ & $\begin{array}{l}\text { Médiation/ } \\
\text { Conciliation }\end{array}$ & $\begin{array}{c}\text { Code de } \\
\text { procédure } \\
\text { pénale } \\
\text { (C.P.P.) }\end{array}$ & Autres & Total* \\
\hline $\begin{array}{l}1991- \\
1992\end{array}$ & 6686 & 2653 & 222 & 1232 & 191 & 10984 \\
\hline $\begin{array}{l}1992- \\
1993\end{array}$ & 7594 & 3077 & 181 & 1304 & 350 & 12506 \\
\hline $\begin{array}{l}1993- \\
1994\end{array}$ & 7975 & 3255 & 418 & 1473 & 268 & 13389 \\
\hline $\begin{array}{l}1994- \\
1995\end{array}$ & 8207 & 3588 & 304 & 849 & 449 & 13397 \\
\hline $\begin{array}{l}1995- \\
1996\end{array}$ & 8908 & 4024 & 219 & 1521 & 548 & 15280 \\
\hline $\begin{array}{l}1996- \\
1997\end{array}$ & 8651 & 3852 & 508 & 1719 & 489 & 15219 \\
\hline $\begin{array}{c}1997- \\
1998\end{array}$ & 8279 & 3734 & 744 & 1622 & 512 & 14891 \\
\hline
\end{tabular}

^ Il est à noter que les jeunes doivent parfois effectuer plus d'une mesure. C'est pourquoi le total de mesures est supérieur au nombre de jeunes accueillis (voir tableau 1).

(entre 1,43\% et 3,34\%) de l'ensemble des mesures administrées par l'ensemble des organismes. Les délégués à la jeunesse ont longtemps hésité à confier l'administration de cette mesure aux OJA, préférant développer eux-mêmes des programmes de médiation. En pratique, cela n'a pas été le cas. Depuis quelques années, les OJA s'emploient à faire valoir leur compétence en ce domaine afin de convaincre les autorités de leur confier la gestion de cette mesure. Peu à peu, ces efforts commencent à porter fruits. On remarque ainsi qu'il s'est tenu 744 mesures de médiation (ou de conciliation) en 1997-1998 (soit $5 \%$ du total des mesures).

S'il est généralement admis que le Québec se distingue du reste du Canada par l'efficacité de son programme de mesures de rechange, ce trait ne semble pas se vérifier en matière de médiation. Ainsi, selon Umbreit (1996), il y aurait eu 574 médiations en 1993 dans les villes de Calgary, Langley, Ottawa et Winnipeg. À elle seule, Winnipeg en compte 327. En comparaison, l'ensemble des OJA du Québec ont encadré seulement 181 jeunes en 1992-1993. Comme l'ont d'ailleurs 
déjà fait remarquer certains criminologues, le Québec fait donc figure de parent pauvre en ce domaine :

Il demeure frappant que, malgré l'insistance qu'elles se sont vu accorder dans de nombreux forums, au cours des dernières années, les mesures liées aux victimes (indemnisation, restitution, remboursement) n'aient été retenues par le Tribunal que dans un cas sur 50, arrivant en fin de liste tout juste derrière les libérations conditionnelles (LaflammeCusson, Langelier-Biron et Trépanier, 1992 : 143).

\section{Pourquoi ce retard?}

Le retard du Québec en ce qui concerne le développement de la médiation entre jeunes contrevenants et leurs victimes a déjà été souligné (Tremblay, 1994 ; Groupe de travail chargé d'étudier l'application de la Loi sur les jeunes contrevenants au Québec, 1995). Sans prétendre pouvoir expliquer de façon définitive ce phénomène, nous voudrions présenter ici un certain nombre de pistes d'explications. Différents facteurs d'ordre institutionnel et professionnel peuvent être identifiés.

Dans un article portant sur la place des victimes dans la justice des mineurs, Cousineau et Tremblay (1996) se penchent sur cette question. Selon ces auteures, le mandat que reçoivent les délégués à la jeunesse est difficilement conciliable avec le développement d'une préoccupation à l'égard des victimes d'actes criminels. Les délégués estimeraient que les victimes sont difficiles d'approche et peu enclines à vouloir rencontrer le contrevenant en cause. Enfin, la définition de leurs tâches les amènerait à concentrer leur attention essentiellement sur les jeunes.

La logique de fonctionnement de certaines institutions pour jeunes peut parfois se traduire en résistances ouvertes à des changements. L'exemple français en témoigne bien. Ainsi, selon Jacques Faget (1997b), il est généralement admis que les innovations en matière de politiques pénales trouvent d'abord une niche dans le secteur de la justice des mineurs, pour ensuite être adoptées par le système judiciaire adulte. L'auteur observe que c'est exactement le contraire qui s'est produit en France en ce qui a trait à la médiation qui a connu un développement plus rapide dans le secteur de la justice des adultes. Selon Faget, ce décalage se rattache ici à un conflit qui perdure depuis les années 1970 et où s'affrontent les perspectives éducatives (ou protectionnelles) et judiciaires. Tout se passe ici comme si la médiation avait été perçue comme une tentative pour minimiser les principes sur lesquels s'appuient de longue date les pratiques des institutions pour jeunes, d'où une résistance face à ce mode d'intervention. 
L'expérience française rejoint donc les éléments d'analyse proposés par certains auteurs québécois (Laflamme-Cusson, Langelier-Biron et Trépanier, 1992 ; Tremblay, 1994 ; Cousineau et Tremblay, 1996) pour expliquer le retard du Québec en matière de médiation. Les résistances des intervenants et des institutions du secteur de la justice pour mineurs semblent ici déterminantes. Ce n'est peut-être donc pas tant la pertinence de cette mesure qui pose un problème que son application dans les centres jeunesse.

D'ailleurs, la médiation est un processus qui connaît une popularité croissante dans d'autres secteurs d'intervention. En témoignent assez bien l'essor des programmes de médiation par les pairs en milieu scolaire, comme « Vers le pacifique » (Centre Mariebourg, 1997), et les initiatives qui débutent en matière de médiation de quartier, projets pouvant s'apparenter aux Maisons de justice et aux Boutiques de droit en France (Bonafe-Schmitt, 1992 ; Wyvekens, 1997). Le déploiement rapide de ces projets, associé à la consolidation de la médiation familiale et à ce qui se fait dans les domaines civil et commercial (Clavier et al., 1997) permettent d'envisager le développement de la médiation dans le champ pénal avec optimisme. À cet égard, les initiatives qui sont mises sur pied en dehors des circuits officiels, de même que les dernières orientations du ministère de la Justice du Québec dans son plan d'action pour 1998-2001 ${ }^{6}$, donnent à croire que le Québec pourra très rapidement rattraper le retard qu'il a pris en ce domaine.

\section{L'évolution des pratiques de médiation dans les 0JA}

La médiation en matière de justice pénale est donc une procédure relativement jeune au Québec. Il n'en demeure pas moins que les pratiques de médiation développées par les OJA ont évolué depuis la mise en place du programme «Entente » par l'équipe du YMCA de Montréal ${ }^{7}$ (Chamberlain 1987) en passant par les programmes de Pivo Jeunesse (TroisRivières) et de Trio Jeunesse (Gatineau). Au gré des critiques et des débats qui ont vu le jour depuis une quinzaine d'années, le sens précis de cette

6. Selon un bulletin d'information du ministère de la Justice, le gouvernement entend bien au cours des prochaines années simplifier et moderniser le système judiciaire, favoriser les modes extrajudiciaires de résolution des conflits, accroître la confiance des citoyens et finalement favoriser une justice réparatrice, moins conflictuelle et adaptée aux réalités socioéconomiques de notre époque. (L’heure juste, édition spéciale, avril 1998, p. 2)

7. Cette équipe fait partie des premiers projets s'apparentant aux OJA. 
mesure n'a pas nécessairement suivi une évolution linéaire, amenant l'ensemble des organismes à revoir collectivement leur approche ${ }^{8}$.

Il est difficile de préciser quelles étaient les orientations précises données à la médiation dans ces organismes. Fruit le plus souvent d'initiatives individuelles, la médiation ne constitue pas alors une pratique formalisée autour de balises bien précises. Il appert en effet que les premières formations en médiation n'ont été offertes qu'en 1986, et ce sous la responsabilité d'organismes communautaires qui adhéraient à un programme élaboré aux États-Unis (Université du Massachusetts). Ces formations s'adressaient alors autant au personnel des centres jeunesse qu'aux intervenants des organismes de justice alternative.

Certains documents permettent toutefois de cerner les principaux objectifs associés à cette mesure. Le Guide d'entrânement pour médiateurs (Peachey, Snyder et Teichroeb, rédigé vraisemblablement en 1982) du programme « Entente » est révélateur des intentions des promoteurs à l'égard de cette mesure et de sa pertinence en matière pénale. S'inscrivant dans une perspective où l'objectif est de réduire les maux produits par le processus pénal, qualifié de contradictoire ou adversatif (créant plus de distance entre les parties plutôt qu'un rapprochement), les auteurs de ce guide estiment que la médiation constitue une démarche susceptible d'harmoniser les rapports entre citoyens en amenant ces derniers à trouver ensemble des solutions à leurs différends. Il est en effet clairement stipulé (section 2.4.3) que la solution au conflit des parties relève de leur propre responsabilité. On précise en outre que les objectifs de la rencontre sont fixés lors de la première étape de la médiation et que cette démarche ne doit pas s'apparenter à un processus visant à sensibiliser le jeune ou à réaffirmer les normes sociales. Les auteurs s'inscrivent donc de plain-pied dans une perspective de justice réparatrice.

Ce guide ne fait d'ailleurs nullement mention « d'infraction », préférant parler en termes de conflit entre deux personnes. On y fait état de la nécessité de modifier le rôle des victimes et celui des contrevenants dans le système de justice, et ce afin de leur redonner la possibilité de résoudre leur conflit. Ce texte est donc porteur d'un discours visant une transformation importante du système pénal. On sait malheureusement peu de choses sur ce programme, si ce n'est que le nombre de médiations

8. En plus de jouer un rôle de précurseurs, les OJA de Trois-Rivières et de Gatineau ont souvent été à l'origine de débats et de questionnements, faisant profiter les autres organismes de leur expérience. 
effectuées par cette équipe a fléchi très rapidement, tant et si bien que cette pratique a pratiquement cessé vers la fin des années 1980 .

Deux autres OJA ont alors commencé à offrir un programme de médiation. Un examen des différents guides de médiation produits par ces organismes (ceux de Trois-Rivières et de Gatineau) permet de dégager certaines convergences dans les pratiques (Trio Jeunesse, 1991 ; Pivo Jeunesse, 1995). En dépit d'une réelle préoccupation à l'égard des victimes, il est clair que l'orientation préconisée par ces deux OJA a principalement pour objectif d'éduquer le jeune auteur d'une infraction. La médiation s'inscrit dès lors dans une logique proche du modèle protectionnel, lequel recouvre l'ensemble des interventions auprès des jeunes contrevenants. L'insertion de la médiation dans le cadre d'un programme de mesures de rechange ne fait somme toute que reproduire une forme d'intervention propre au système pénal. Ainsi, les rencontres préalables servent ici davantage à expliquer le processus pénal que le déroulement même de la médiation. Bien que visant à favoriser une réparation des torts subis, la médiation a également pour objectif d'amener les jeunes à prendre conscience des conséquences que leurs gestes ont entraînées. En comparaison du programme "Entente », on constate donc un glissement de perspective au chapitre des objectifs de la médiation. Avec les critiques qui commencent à se faire entendre et alimentés par les débats sur la médiation, certains ajustements s'imposeront assez rapidement.

\section{Les critiques}

Depuis la mise sur pied de programmes de médiation (qu'ils soient le fait des OJA ou des centres jeunesse), de nombreux intervenants se sont exprimés sur cette mesure, n'hésitant pas à critiquer les objectifs mis de l'avant et ses finalités (Groupe de travail chargé d'étudier l'application de la Loi sur les jeunes contrevenants au Québec, 1995 ; Cousineau et Tremblay, 1996; Charbonneau, 1998a). Dans un mémoire présenté en mai 1987, Plaidoyer-victimes, un organisme de défense des droits des victimes d'actes criminels, souligne ainsi que "sous des allures avant-gardistes, la Loi sur les jeunes contrevenants n'a pas sensiblement modifié le statut de la victime » :

On demande en fait à la victime souvent accablée de polytraumatismes de consentir un petit effort supplémentaire pour venir aider un jeune à prendre conscience de ses responsabilités ; la victime devient alors un instrument pédagogique. C'est le reproche qui a été fait aux délégués à la jeunesse par les services d'aide aux victimes (Lajoie, 1987 : 5). 
Plus récemment, Walgrave (1993) a attiré l'attention sur les risques de voir la pratique de la médiation être détournée de ses intentions premières au profit d'objectifs d'éducation ou de réhabilitation du jeune contrevenant. Au total, ces différentes critiques ont donné un nouveau souffle à la réflexion entourant la pratique de la médiation. Elles ont ainsi conduit les principaux responsables de ces programmes à revoir leurs manières de faire. En 1996, les organismes de Trois-Rivières et de Gatineau, de concert avec le Regroupement des organismes de justice alternative du Québec, ont décidé de mettre en commun leur expertise afin de rédiger un Guide de médiation (ROJAQ, 1996) et d'en faire un outil de référence pour l'ensemble des OJA du Québec. Répondant aux principales critiques, ce guide visait notamment à faire du processus de médiation un mécanisme au sein duquel les préoccupations des victimes et celles des contrevenants ont une même importance.

\section{Discours de légitimation et critiques}

Alors que les OJA discutent encore du véritable sens de la justice réparatrice et des pratiques de médiation à privilégier, nombre d'auteurs américains ont initié un débat portant sur les objectifs de la médiation, débat introduisant des éléments qui nous semblent nouveaux. Pour Bush et Folger (1994), les techniques de médiation en matière criminelle sont généralement conçues dans l'optique de satisfaire les parties et de promouvoir une plus grande justice sociale. Or, selon ces derniers, la principale motivation qui devrait animer les médiateurs réside dans le pouvoir de la médiation à transformer les individus. Ces auteurs introduisent ici une typologie qui résume assez bien les principaux arguments avancés au cours des dernières années pour légitimer ou décrier la pratique de la médiation. Selon eux, il existe trois types d'argumentation servant à justifier le recours à la médiation : 1) la satisfaction ; 2) la justice sociale ; 3) la transformation des individus. Ils complètent cette typologie en présentant une dernière argumentation qui dénonce ce type de démarche : 4) l'oppression.

Le discours axé sur la médiation comme source de satisfaction valorise une telle démarche pour ses capacités à fournir aux parties impliquées une occasion de traiter tous les aspects de leur différend. Ici, c'est le résultat qui compte, le processus devant conduire à une entente dite « gagnant-gagnant ». En favorisant le rapprochement des parties, cette entente réduit les tensions entre individus et se distingue de l'approche adversative ou contradictoire basée sur le marchandage et la négociation. Les promoteurs d'une telle approche insistent plus particulièrement 
sur le fait que la médiation permet d'importantes économies, tout en réduisant les coûts sociaux associés aux conflits. Parmi les auteurs associés à cette tendance, on retrouve notamment Fisher et Ury (1982), connus pour leurs travaux sur la négociation raisonnée.

Le second discours s'inscrit davantage dans une perspective de transformation sociale. Les promoteurs de la médiation comme outil de justice sociale souhaitent en effet redonner plus de pouvoir aux communautés dans la gestion des problèmes sociaux et réduire leur dépendance à l'égard des institutions publiques. Il s'agit pour eux d'amener les citoyens à se réapproprier un pouvoir sur leur vie tout en développant une plus grande cohésion communautaire. Plusieurs projets et initiatives mettent de l'avant une telle forme de justification : c'est le cas des comités de médiation de quartier (très répandus aux États-Unis) ainsi que des Boutiques de droit en France, aujourd'hui reprises sous l'appellation « Maison de justice » (Wyvekens, 1997).

Le troisième type de discours de légitimation s'articule autour de l'idée voulant que le processus de médiation constitue un moyen pour transformer les individus. Selon les promoteurs d'une telle approche, la médiation doit contribuer à sensibiliser les deux parties impliquées aux réalités vécues par l'autre tout en les amenant à développer leur empathie, leur compassion et leur capacité à gérer un conflit. La médiation apparaît ici comme un outil dont la finalité première est donc de rendre les gens meilleurs en misant en quelque sorte sur leur « humanisme » (Umbreit, 1997). Partisans d'une telle approche, Bush et Folger (1994) font en outre valoir qu'une telle perspective implique un changement fondamental dans la manière de conduire une médiation. Cette question a fait l'objet d'une réflexion intéressante, où Umbreit (1997) établit des distinctions entre les techniques d'une médiation « humaniste » (visant la transformation des individus) et les techniques d'une médiation traditionnelle.

Se démarquant de ces argumentations, certains auteurs estiment que la médiation contribue à l'oppression d'une des parties. Ces derniers font valoir qu'une telle démarche ne fait que réduire la capacité des gens à reprendre le contrôle sur leurs difficultés. La neutralité du médiateur est plus particulièrement pointée, ce statut pouvant conduire certains agresseurs à abuser de leur force ou de leur pouvoir en toute impunité. Qui plus est, la médiation comporterait des effets pervers en ce qu'elle peut parfois amener un individu à ne pas se prévaloir de ses droits et à ne pas inscrire une action légale contre son agresseur. De telles critiques sont généralement formulées par les groupes de défense des droits de victimes. D'ailleurs, dans la mesure où la question de la violence conjugale demeure un sujet très 
délicat, les partisans de la médiation familiale préferrent généralement condamner l'utilisation de la médiation dans de tels cas (Lévesque, 1998).

En somme, la typologie proposée par Bush et Folger s'avère très utile en ce qu'elle fournit des repères permettant de revoir les pratiques et de les adapter afin que ce processus ne se limite pas à une simple négociation des dédommagements matériels. Elle permet également de jeter un regard critique sur l'évolution des pratiques de médiation dans les OJA. Si les premiers organismes voyaient dans la médiation un moyen de participer à des changements sociaux, force est de constater, avec le recul, que certaines pratiques se sont cantonnées dans une technicalité peu propice à de tels changements. En outre, plutôt que d'être axées vers la quête de la satisfaction des parties, certaines expériences s'inscrivaient souvent dans un courant proche de la justice réhabilitative, et ce, au risque de participer à « l'oppression » des victimes alors utilisées comme outil thérapeutique pour le traitement des jeunes contrevenants. À cet égard, une telle critique sied autant aux OJA qu'aux centres jeunesse.

En septembre 1998, les OJA se sont donné un nouveau Guide de médiation (ROJAQ 1998). Les rédacteurs de ce guide avaient notamment pour but de fournir aux OJA un outil permettant de rediriger les pratiques vers des objectifs propres au modèle de justice réparatrice, en tentant de réduire la prédominance du caractère punitif et protectionnel des interventions antérieures. Ainsi, une attention toute particulière a été apportée afin de mettre en place des procédures susceptibles de garantir une plus grande impartialité. Ce guide propose certaines dispositions visant à donner à la médiation des objectifs adaptés autant aux contrevenants qu'aux victimes. Les OJA sont invités à recourir à des médiateurs bénévoles, le rôle des intervenants se limitant à convoquer et à préparer les parties. Une telle démarche vise à redonner aux participants le plein contrôle sur l'issue de la médiation.

À la lumière de la typologie développée ci-haut, ce nouveau guide (ROJAQ, 1998) ne doit pas être perçu comme une simple tentative pour expurger tout l'aspect relatif à l'éducation (ou à la transformation) des jeunes et des victimes. En fait, le débat reste à faire au Québec sur la question des rapprochements possibles entre l'approche voulant faire de la médiation une source de transformation des individus et la perspective réhabilitative en justice (Walgrave, 1993). Sans rejeter d'emblée l'idée voulant que le processus de médiation ait un impact positif pour les individus, certaines intentions associées à la médiation doivent faire l'objet d'une attention particulière, ne serait-ce par exemple que la question du pouvoir magique de la médiation de même que toute la dimension spirituelle qui l'accompagne 
(Bush et Folger, 1994 ; Umbreit, 1998). Ces débats, et les nuances qu'ils introduisent, risquent de ranimer des discussions qui avaient cours au Québec au moment de la Révolution tranquille. En effet, alors que nous vivons depuis peu la déconfessionnalisation de nos écoles ${ }^{9}$, de nombreux auteurs conferent à la médiation un pouvoir presque mystique.

Attribuer au processus de médiation des objectifs propres à transformer (dans le sens de rééduquer) les individus repose sur des prémisses douteuses. Nous ne pouvons souscrire à une approche qui, poussée à l'extrême, aurait pour objectif de réduire la dimension « diabolique » de la nature humaine pour y faire triompher la «bonté » ou une forme «d'humanisme ». Au risque de caricaturer quelque peu ce mouvement, nous oserions dire que nous ne sommes pas très loin d'un Vade retro Satana ${ }^{10}$.

Sans nier les dimensions spirituelles de la médiation, il ne nous apparaît pas souhaitable d'en faire la justification première de son développement. En dépit du respect que l'on peut porter à ces dimensions, celles-ci ne sauraient en aucun cas être érigées au rang d'objectif principal de la médiation. Juxtaposées aux contributions d'auteurs tel que Walgrave (1993), ces réflexions témoignent bien de l'importance de scruter plus à fond les différents discours autour de la médiation et d'en entreprendre une nouvelle lecture afin de statuer sur leur pertinence respective.

En tout état de cause, les prochains développements de la médiation au Québec devront s'accompagner de mécanismes d'évaluation. Au Québec, les recherches en ce domaine font encore cruellement défaut. Il importe de développer des outils permettant d'évaluer les résultats de la médiation autour de variables aussi fondamentales que la satisfaction des parties, leur impression quant à l'équité du processus et leur sentiment de justice. De telles études pourraient en outre être utiles afin de vérifier si les situations qui font l'objet d'une médiation n'ont pas tout simplement pour effet d'élargir un peu plus le filet du contrôle social (Cohen, 1985) ${ }^{11}$.

9. Jusqu'à tout récemment encore, les commissions scolaires publiques du Québec étaient d'orientation catholique ou protestante.

Io. Pour Bush et Folger (1994 : 29-30), il est clair « That the goal of transformation that is, engendering moral growth toward both strenght and compassion - should take precedence over the other goals of mediation [...]». Selon ces derniers, «We have within us the potential for positive and negative, good and evil, bigher and lower, buman and unbuman, and the ability to know the difference ». C'est nous qui mettons l'italique.

II. Ce danger est bien présent comme le soulignent Mary et De Fraene (1998) : «Le travail d'intérêt général et la médiation pénale ne sont donc pas seulement les éventuels signes avant-coureurs d'une nouvelle justice pénale réparatrice ou restauratrice, mais surtout les instruments d'une repénalisation de facto de la petite délinquance, dans le cadre d'une justice pénale qui reste fondamentalement rétributive (sic). » 


\section{Conclusion}

Les premières expériences québécoises de médiation en matière criminelle se sont donc développées à la fin des années 1970 dans le secteur de la justice des mineurs. L'essor de la médiation s'est toutefois heurté à plusieurs formes de résistances et a donné lieu au déploiement de manières de faire très différentes, pratiques soutenues par des discours à l'occasion contradictoires. Pendant ce temps, les expériences de médiation à l'extérieur du Québec ont connu un développement impressionnant. Après vingt ans d'expérimentation, les débats sur le sens à donner à ces expériences sont loin d'être terminés. Il appartient maintenant aux acteurs québécois de profiter de ces discussions et de procéder à l'évaluation de ce qui se fait, et ce, afin de développer une perspective adaptée aux réalités d'ici et de permettre l'expérimentation de pratiques sociales porteuses de changement. Il apparaît en outre essentiel que la pratique de la médiation puisse déborder le champ de la justice des mineurs pour se faire une place dans ce qu'il est convenu de nommer le champ de la justice des adultes.

\section{Références}

Association des services de réhabilitation sociale du Québec (ASRSQ), (1998), «Dossier : la justice réparatrice », Porte ouverte ( $\mathrm{n}^{\circ}$ spécial), vol. $\mathrm{X}, \mathrm{n}^{\circ} 1$.

BonAFE-SchmitT, J.P. (1992), «Les Boutiques de droit, l'autre médiation », Archives de politique criminelle, $\mathrm{n}^{\circ} 14$, p. 57-70.

Bush, R. et Folger, J. (1994), The Promise of Mediation, San Francisco, Jossey Bass Publishers.

Centre Mariebourg, (1997), Vers le pacifique, Montréal, Centre Mariebourg.

Chamberlain, L. (1987), La conciliation dans le système de justice pour jeunes, mémoire de maîtrise, Université d'Ottawa.

Charbonneau, S. (1998a), « La justice réparatrice au Québec », conférence prononcée dans le cadre de la Journée de sensibilisation sur la justice réparatrice, organisée par la Fondation «Le Parrain », (12 février).

Charbonneau, S. (1998b), «Restorative Justice Trajectory in Quebec » in Walgrave, L. (dir.), Restorative Justice for Juveniles. Potentialities, Risks and Problems, Leuven, Leuven University Press, p. 229-243.

Clavier, H. et al. (1997), Guide pratique de médiation, Toronto, Carswell.

Cohen, S. (1985), Visions of Social Control : Crime, Punishment and Classification, Cambridge, Polity.

Commission de réforme du droit (CRD) (1976), La participation communautaire à la réadaptation du délinquant, Ottawa, Approvisionnements et services Canada. 
Cousineau, m.m. et Tremblay, a. (1996), « jeunes contrevenants et mesures de réparation : entre la lettre de la loi et son application », in COITEux j. et al (dir.), Question d'Équité. l'aide aux victimes d'actes criminels, montréal, association québécoise plaidoyer-victimes, p. 157-180.

D'Amours, O. (1982), «Survol historique de la protection de l'enfance au Québec de 1608 à $1977 »$, annexe 1 au rapport de la Commission parlementaire spéciale sur la protection de la jeunesse.

DESDEVISES, M.C. (1993), «L'évaluation des expériences de médiation entre délinquants et victimes : l'exemple britannique », Revue de science criminelle et de droit pénal comparée, $\mathrm{n}^{\circ} 1$, p. $45-62$.

FAGET, J. (1997a), La médiation. Essai de politique pénale, Paris, Éd. Erès.

FAGET, J. (1997b), « Le cadre juridique et éthique de la médiation pénale », in La médiation pénale. Entre répression et réparation, Paris, L'Harmattan, p. 35-54.

FISHER, R. et URY, W. (1982), Comment réussir une négociation, Paris, Seuil.

Groupe de travail chargé d'étudier l'application de la Loi sur les jeunes contrevenants au Québec (1995), Les jeunes contrevenants : au nom ... et au-delà de la loi, Québec, ministère de la Santé et Services sociaux et ministère de la Justice.

Laflamme - Cusson, S., Langelier - Biron, L. et Trépanier, J. (1992), La prise de décision à l'égard des jeunes contrevenants, Montréal, Centre international de criminologie comparée, Université de Montréal.

LAJOIE, J. (1979), Projet d'intervention jeunesse, rapport final, Bureau de consultation jeunesse inc., Montréal, inédit.

LAJOIE, J. (1987), La place de la victime dans notre système de justice pénale juvénile ou les droits de la victime à la merci des autres, mémoire présenté dans le cadre de la consultation sur les victimes d'actes criminels, Montréal, inédit.

LEROux, F. (1997), Justice réparatrice et délinquance juvénile: une analyse pluridisciplinaire de l'adéquation de la théorie réparatrice à la délinquance des jeunes, mémoire présenté en vue de l'obtention du grade de licencié en psychologie, Louvain-la-Neuve.

LÉvesque, J. (1998), Méthodologie de la médiation familiale, Saint-Hyacinthe, Edisem.

MARY, P. et De FraEne D. (1998), Sanctions et mesures dans la communauté. État critique de la question en Belgique, Bruxelles, Université libre de Bruxelles.

Ministère de la Justice (1998), L’heure juste, édition spéciale.

Peachey, D.E., Snyder, B. et Teichroeb, A. (vers 1982), Guide d'entraînement pour médiateurs au sein du système de justice criminelle, Montréal, projet « Entente».

Pivo Jeunesse (1995), Guide de formation (technique de médiation), Trois-Rivières, inédit.

Regroupement des organismes de justice alternative du Québec (ROJAQ) (1996), Guide de médiation, Montréal, inédit. 
Regroupement des organismes de justice alternative du Québec (ROJAQ) (1998), Guide de médiation en matière criminelle, Montréal, inédit.

Solliciteur général du Canada (1982), La Loi sur les jeunes contrevenants, 1982. Points saillants, Ottawa, Division des communications, Direction des programmes, Approvisionnements et services Canada.

TRemblay, A. (1994), Justice des mineurs: quand la victime a voix au chapitre, mémoire de maîtrise, Université de Montréal.

TRÉPANIER, J. (1988), Le contrôle de la délinquance juvénile par la recherche de ses causes et par la protection de l'enfance, Université catholique de Louvain, département de criminologie.

TrÉPANIER, J. et TulKens, F. (1995), Délinquance et protection de la jeunesse : aux sources des lois belge et canadienne sur l'enfance, Montréal et Bruxelles, Presses de l'Université de Montréal.

Trio jeunesse (1991), Guide de procédures conciliation, Hull, inédit.

UMBreIt, M. (1995), Mediating Interpersonnal Conflicts : A Pathway to Peace, West Concord (MN), CPI Publishing.

UMBreIT, M. (1996), « Restorative Justice Througt Mediation : The Impact of Programs in Four Canadian Provinces », in GaLawaY, B. et HudSON, J. (dir.), Restorative Justice: International Perspective, Monsey (NY), Criminal Justice Press.

UMBReIT, M. (1997), « Humanistic Mediation : a Transformative Journey of Peacemaking ", Mediation Quaterly, vol. 14, n ${ }^{\circ}$ 3, p. 201-213.

UMBreit, M. (1998), "Victim-Offender Mediation in Cases of Severe Violence », conférence présentée dans le cadre de la Second Annual International Conference on Restorative Justice for Juveniles, Fort Lauderdale, (7-9 novembre).

Umbreit, M. et Greenwood, J. (1998), National Survey of Victim Offender Mediation Programs in the US, Center for Restorative Justice \& Mediation, Inédit.

WALGRAVE, L. (1993), « La justice réparatrice et les jeunes », in GAZEAU, J.F. et PEYRE, V. (dir.), Au-delà de la rétribution et de la rébabilitation : la réparation comme paradigme dominant dans l'intervention judiciaire contre la délinquance des jeunes,

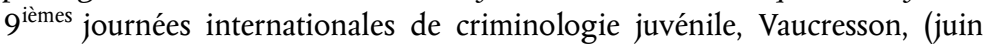
1993).

WRIGHT, M. (1996), Justice for Victims and Offenders : A Restorative Response to Crime, Winchester, Waterside Press.

WYVEKENS, J. (1997), «Les maisons de justice : sous la médiation, quelle troisième voie? », in La médiation pénale. Entre pression et réparation, Paris, L'Harmattan, p. 61-81.

Zenr, H.J. (1990), Changing Lenses : A New Focus for Crime and Justice, Scott Dale (PA) et Waterloo (Ont.), Herald Press. 
